\title{
Causes, consequences, and reversal of immune system aging
}

\author{
Encarnacion Montecino-Rodriguez, Beata Berent-Maoz, and Kenneth Dorshkind
}

Department of Pathology and Laboratory Medicine, David Geffen School of Medicine at UCLA, Los Angeles, California, USA.

\begin{abstract}
The effects of aging on the immune system are manifest at multiple levels that include reduced production of $B$ and $T$ cells in bone marrow and thymus and diminished function of mature lymphocytes in secondary lymphoid tissues. As a result, elderly individuals do not respond to immune challenge as robustly as the young. An important goal of aging research is to define the cellular changes that occur in the immune system and the molecular events that underlie them. Considerable progress has been made in this regard, and this information has provided the rationale for clinical trials to rejuvenate the aging immune system.
\end{abstract}

\section{Introduction}

One of the most recognized consequences of aging is a decline in immune function. While elderly individuals are by no means immunodeficient, they often do not respond efficiently to novel or previously encountered antigens. This is illustrated by increased vulnerability of individuals 70 years of age and older to influenza (1), a situation that is exacerbated by their poor response to vaccination (2-4).

The effects of aging on the immune system are widespread and affect the rate at which naive $B$ and $T$ cells are produced as well as the composition and quality of the mature lymphocyte pool. The goal of this article is to review recent advances, with a focus on adaptive immunity, in the understanding of the cellular and molecular events underlying these age-induced alterations and discuss their implications for the design of strategies to rejuvenate the immune system in the elderly.

\section{Effects of aging on immune system development}

Following their production in the bone marrow and thymus, naive $\mathrm{B}$ and $\mathrm{T}$ cells migrate to secondary lymphoid tissues such as the spleen (5-7). This process is particularly robust in the young in order to generate a diverse immune repertoire and to fill peripheral lymphoid compartments. In contrast, primary lymphopoiesis in the elderly is significantly diminished, as exemplified by involution of the thymus $(8-10)$. The causes of this age-related reduction in lymphocyte development are multifactorial and include changes in HSCs and progenitor cells $(11,12)$ as well as the local tissue and systemic environments $(13,14)$.

HSCs exhibit multiple age-related changes that include impaired adherence to stromal cells and, in some strains of mice $(15,16)$ and elderly humans (17), an increase in number. From an immunologic perspective, the most profound effect of stem cell aging in both mice $(11,18)$ and humans $(17)$ is a decreased capacity to produce lymphocytes and an increase in myeloid potential. This shift has been correlated with increased expression of myeloid lineage genes and downregulation of those specifying a lymphoid lineage fate $(11,17)$.

The ability to identify, at least in mice, distinct HSC subsets that are lymphoid biased or myeloid biased, or that exhibit balanced lympho-myeloid potential has provided new insights into how aging affects the stem cell pool (Figure 1 and refs. 19, 20). Because

Conflict of interest: The authors have declared that no conflict of interest exists. Citation for this article: J Clin Invest. 2013;123(3):958-965. doi:10.1172/JCI64096. each of these lineage-negative $\left(\mathrm{Lin}^{-}\right) \mathrm{CD} 117^{+}\left(\mathrm{c}-\mathrm{kit}{ }^{+}\right) \mathrm{Sca}-1^{+}$populations can be defined based on their relative expression of CD150, a signaling lymphocytic activation molecule family member (Figure 1 and refs. 21-23), it is now appreciated that the number of lymphoid-biased stem cells declines with age concomitant with an increase in myeloid-biased HSCs (19). The age-related increase in expression of myeloid lineage genes in the studies noted above (11, 17) likely resulted from the accumulation of myeloid-biased HSCs, as these analyses were performed on unseparated pools of HSCs. Despite the increase in their number, myeloid-biased stem cells are not as robust as their young counterparts (24), which in turn could underlie the numerous age-related deficiencies observed in mature myeloid cells such as neutrophils and macrophages $(25,26)$.

Lymphoid-biased HSCs in old mice also appear to accumulate deficiencies that, as discussed below, may compromise their selfrenewal potential and contribute to the decline of this population (27). In view of this, it is not surprising that the number of B cell $(28-31)$ and $\mathrm{T}$ cell $(32,33)$ progenitors in bone marrow and thymus is markedly reduced with age. In addition, the poor quality of the lymphoid progenitors that are generated in the aged further exacerbates the decline in lymphopoiesis $(29,32,34)$. For example, common lymphoid progenitors, pre-pro-B cells, and pro-B cells from old mice do not proliferate as extensively as do young cells, and they exhibit significantly higher rates of apoptosis. The most immature intrathymic progenitors, which have been termed early T lineage progenitors (ETPs), generate $\mathrm{CD}^{-}{ }^{-} \mathrm{CD} 8^{-}$double-negative $(\mathrm{DN})$ progeny that ultimately become mature $\mathrm{CD} 4^{+}$Th cells and $\mathrm{CD}^{+}$cytotoxic T cells (35). Similar to B cell progenitors, ETPs and DN cells from old mice also exhibit age-related defects in proliferation and high rates of apoptosis (ref. 32 and Figure 1).

Why aging results in a decline in the number of lymphoid-biased HSCs and reduced quality of lymphoid progenitors is not fully understood. On the one hand, intrinsically programmed events in stem and progenitor cells may be operative, which would presume that these cells have some sort of internal clock that regulates their function and longevity. However, accumulating evidence suggests that declines in lymphopoiesis are influenced by age-related changes in the environment. The precise, age-related environmental factors that result in depletion of lymphoid-biased HSCs have not been identified, although changes in levels of transforming growth factor $\beta-1$ might be involved (21). Declines in the bone marrow microenvironment, possibly as a result of decreased IL-7 secretion by stromal cells, have been implicated in B cell lineage aging $(36,37)$. Age-relat- 


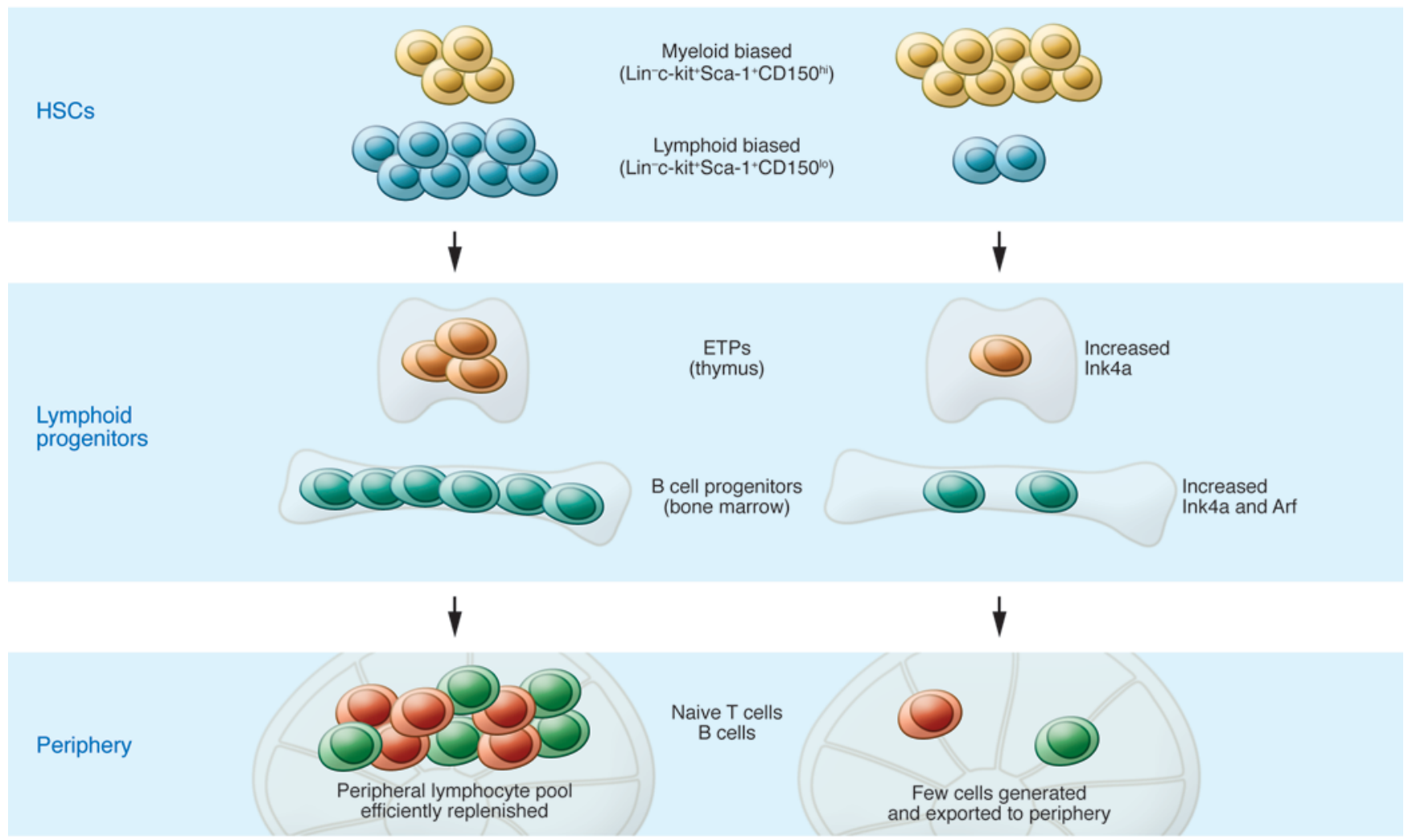

\section{Figure 1}

Effects of aging on HSCs and lymphocyte progenitors. Lymphopoiesis in the young (left) is characterized by robust B and T cell production in the bone marrow and thymus. The pool of HSCs includes a relatively high number of lymphoid-biased stem cells that efficiently generate lymphoid progenitors with high proliferative potential. However, with increasing age (right), the number of lymphoid-biased HSCs declines and myeloidbiased stem cells predominate, contributing to the reduced numbers of lymphoid progenitors. In addition, B cell progenitors in the bone marrow and $\mathrm{T}$ cell progenitors in the thymus exhibit reduced rates of proliferation and higher levels of apoptosis compared with their young counterparts. The increased expression of Ink4a and Arf in pro-B cells and Ink4a in ETPs contribute to this decreased proliferation/increased apoptosis. The decline in primary lymphopoiesis in turn results in a reduced number of naive cells that migrate to secondary lymphoid tissues such as the spleen.

ed microenvironmental changes also have a major impact in the thymus, where T cell development is dependent upon an intact thymic milieu composed of fibroblasts, macrophages, dendritic cells, and thymic epithelial cells. It is clear that the number of thymic epithelial cells declines over time and that they are not replaced, as a result of impaired proliferation (38). In addition, the production of inflammatory mediators that may be thymocytotoxic also increases with age (39). The thymus, particularly in humans, is increasingly infiltrated with adipocytes, the byproducts of which may be toxic to developing thymocytes and to the remaining stromal cell populations (40). A similar fatty deterioration of the bone marrow microenvironment occurs (41), but the role of adipocyte-derived factors in suppression of B lymphopoiesis is not well understood.

Systemic changes that occur during aging may also affect lymphocyte production (14) and thymopoiesis in particular. For example, numerous reports have demonstrated that growth hormone (GH) and IGF-1 can stimulate thymopoiesis $(13,42)$. Because the production of these hormones declines with age (43), this has led to the conclusion that age-related changes in the endocrine system contribute to declines in lymphopoiesis. This view has formed the rationale for clinical trials using $\mathrm{GH}$, which we discuss below.

\section{Aging and the decline of immune function}

Although the number of naive $B$ and $T$ cells that migrate from primary to secondary lymphoid organs is reduced by aging, $\mathrm{B}$ and $\mathrm{T}$ cell development does not cease entirely. Indeed, some functional thymic tissue remains even in elderly humans (44). The continued production of lymphocytes, albeit limited, and the presence of relatively normal numbers of lymphocytes in organs such as the spleen raises the question of why functional immunity declines in the elderly. The answer is that the composition and quality of the mature lymphocyte pool is profoundly altered by aging.

For example, an increase in the number of memory $T$ cells is now a well-recognized feature of aging. These cells, which are generated following the initial encounter with antigen, persist long after the initial challenge has cleared and provide a source of effectors that can respond rapidly upon antigen re-exposure. Exposure to multiple pathogens over time results in a diverse immune repertoire that includes an increased pool of protective memory cells. However, chronic stimulation with persistent viral infections such as CMV can exhaust the naive pool of cells and result in an oligoclonal memory cell expansion. This phenomenon is thought to be a major factor contributing to the accumulation of $\mathrm{CD}^{+}$memory 


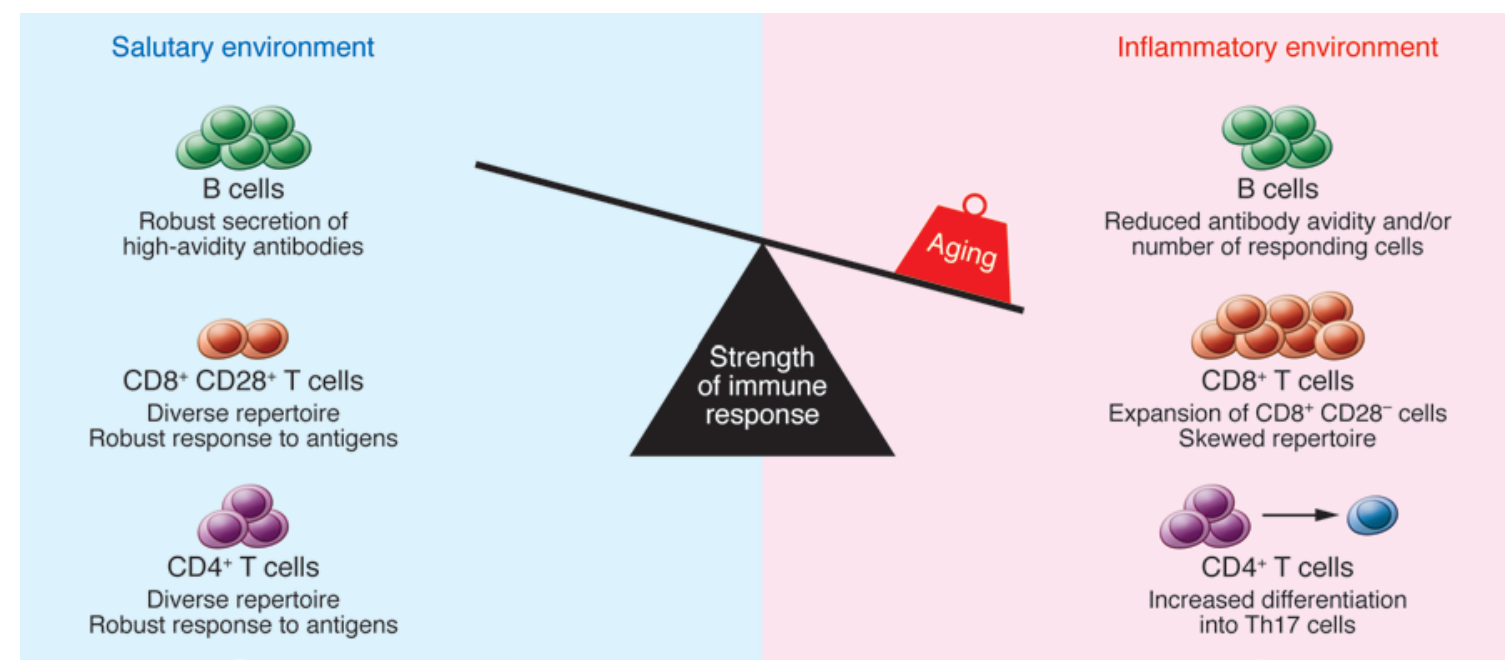

\section{Figure 2}

The strength of the immune response declines with age. Multiple age-related changes can affect the composition and function of lymphocytes in secondary lymphoid tissues. CD4+ Th cells exhibit activation defects and increased differentiation into Th17 cells. CD8 ${ }^{+} \mathrm{T}$ cells undergo an oligoclonal expansion and loss of CD28 in humans and exhibit impaired function. The number of B cells that respond to influenza is reduced, and antibody avidity in response to carbohydrate antigens is diminished. In addition, the tissue environment includes an increased concentration of inflammatory cytokines, which may be produced by stromal elements, dendritic cells, or aging B and T cells. The increased number of memory cells that occupy tissue niches and the inflammatory milieu in turn may compromise the ability of naive B and T cells migrating from the bone marrow and thymus to lodge in the tissue. Together, these changes result in diminished immune function in the elderly.

cells in the elderly (45), although antigen-independent expansion of $\mathrm{CD}^{+} \mathrm{T}$ cells may also occur (46). These oligoclonal expanded $\mathrm{CD}^{+}$memory $\mathrm{T}$ cells are further distinguished in humans by loss of expression of the CD28 co-stimulatory molecule $(47,48)$ and impaired immune function. The accumulation of $\mathrm{CD} 8^{+} \mathrm{CD} 28^{-} \mathrm{T}$ cells and CMV seropositivity are components of the immune risk profile, which has been proposed as a predictor of mortality in individuals 65 and older (49), but precisely how the combination of these events results in increased death is not clear (50).

The immune risk profile also includes B cells with impaired function. B cell number in mice is unchanged by aging, but in human peripheral blood their absolute number is reduced (51). This decline is likely due to decreased numbers of $\operatorname{IgM}^{+}$memory and switched memory B cells, because the total number of naive $B$ cells remains unchanged by aging $(51,52)$. Human and murine B cells also exhibit impaired class switch recombination, which has been attributed to decreased induction of activation-induced cytidine deaminase (AID) enzyme (53). In view of the critical role played by $\mathrm{CD}^{+} \mathrm{T}$ cells in orchestrating the immune response, some of the age-related deficiencies observed in B cells $(51,53,54)$, which include a reduction in the number of plasmablasts that develop in response to influenza vaccination (55) and the production of poor-quality antibodies to carbohydrate antigens (56-58), may occur secondary to $\mathrm{T}$ cell deficiencies.

Multiple B cell subpopulations have been defined, and additional study is required to determine how aging affects them. Thus, humoral immunity to influenza virus occurs in T cell-independent and $\mathrm{T}$ cell-dependent waves that may involve B1, marginal zone, and follicular B cells (59). It was recently reported that marginal zone B cell number is reduced in old mice (60), but whether and how this affects production of antibodies to specific antigens is unknown. Although present at a low frequency (61), B1 B cells play a critical role in the response to $S$. pneumoniae in mice by the secretion of anti-carbohydrate antibodies (62). However, very little is known about the effects of aging on their function. A population of $\mathrm{CD} 20^{+} \mathrm{CD} 27^{+} \mathrm{CD} 43^{+} \mathrm{CD} 70^{-}$human $\mathrm{B} 1$ cells was recently described (63), but again, nothing is known about how aging affects their development or function.

Inflammaging (64), a condition in which there is an accumulation of inflammatory mediators in tissues, has been associated with aging. The source of these inflammatory factors has been proposed to be cells that have acquired a senescence-associated secretory phenotype (SASP) (65). The SASP could be acquired by cells once they have aged, or it may occur gradually in various populations over time as they acquire DNA lesions that in turn trigger the increased production of inflammatory mediators such as IL-6 $(66,67)$. Regardless of how the shift occurs from a salutary to an inflammatory milieu, the end result may be a negative effect on the ability of naive lymphocytes from the bone marrow or thymus to lodge in an organ such as the spleen as well as the function of mature lymphocytes already resident in that tissue. In the latter case, for example, $\mathrm{CD}^{+} \mathrm{T}$ cells that accumulate in aged individuals proliferate poorly $(68,69)$, and, as discussed in more detail in the following section, $\mathrm{CD}^{+} \mathrm{T}$ cells exhibit reduced $\mathrm{T}$ cell receptor signaling intensity and altered production of various cytokines following antigen binding $(70,71)$.

The precise source of the various inflammatory mediators may vary between individuals. In one scenario, age-related changes in microenvironmental elements such as stromal cells or dendritic cells (72) would result in a shift from a salutary to an inflammatory environment. However, it is equally plausible that changes occurring in aging $\mathrm{B}$ and $\mathrm{T}$ cells or innate effectors (26) may alter microenvironmental elements. For example, with increased age, naive $\mathrm{CD}^{+} \mathrm{T}$ cells exhibit biased differentiation into Th17 instead of Th1 and Th2 cells, and the inflammatory mediators secreted by the latter cells could in turn affect stromal cells or other environmental 
Table 1

Potential interventions to rejuvenate the aging immune system

\begin{tabular}{lccc}
\hline Target & Goal & Possible intervention & Reference \\
HSC & Increase function; increase number & Cdc42 inhibitors, antioxidants, BATF inhibitors & $27,76,95,96$ \\
& of lymphoid-biased stem cells & & \\
B cell progenitor & Stimulate B cell lymphopoiesis & Inhibition of & Ink4a or Arf, mature B cell depletion \\
Thymus & Stimulate production of naive T cells & Inhibit Ink4a; GH, IGF-1, and & 81,109 \\
& and export to periphery & FGF7 administration; CR & $89-101,103,105,112$ \\
CD4+ memory T cell & Increase function & Inhibition of DUSP4 & Inhibition of DUSP6 \\
Naive CD4+ T cell & Increase function & Rapamycin & 87 \\
CD8+ T cell & Increase function & Cytotoxic drugs & 110 \\
Aged B and T cell & Remove dysfunctional mature B and T cells & & 109 \\
& to free niches in secondary organs & Anti-inflammatory drugs & \\
Inflammaging & Reduce inflammatory and restore salutary milieu & FGF7 in thymus & 106,107 \\
Hematopoietic & Remove dysfunctional cells and/or & & \\
microenvironment & restore function of existing cells & & \\
\hline
\end{tabular}

Many of the suggested interventions discussed herein have not been evaluated in preclinical models or clinical trials.

populations (73). The development of Th17 cells is dependent on the basic leucine zipper transcription factor ATF-like (BATF) transcription factor (74), but why its expression might increase with age is not clear. Similarly, the $\mathrm{CD}^{+} \mathrm{CD} 28^{-}$memory $\mathrm{T}$ cells that accumulate in aged humans may also be a source of inflammatory cytokines $(68,69)$ that affect the function of stromal elements. The specific cells and the order in which age-related changes occur in them may vary between individuals as a result of differences in genetic background and environmental exposure. However, once the effects of aging are manifest in one or more target populations, a vicious cycle may initiate that leads to a downward spiral of increasingly compromised immune function (Figure 2).

\section{Intracellular changes in developing and mature lymphocytes}

The analysis of stem and progenitor cells from various tissues has revealed that aging often results in the dysregulation of similar molecular pathways (75). For example, alterations in the PI3K pathway are frequently exhibited by aging cells. Consistent with this observation, HSCs are depleted due to build-up of ROS in mice in which FOXO, a key downstream PI3K component, is deleted (76). Levels of ROS in cells may also be regulated by ataxia telangiectasia mutated (ATM) kinase, and age-related declines in expression of ATM can result in an increase in ROS in cells $(77,78)$. Increased expression of tumor suppressor proteins such as $\mathrm{p} 16^{\text {Ink4a }}$ and ARF also occurs in aging cells from multiple tissues $(79,80)$. Expression of $\mathrm{p} 16^{\text {Ink4a }}$ results in activation of retinoblastoma, leading to cell cycle inhibition, while p19 ${ }^{\text {Arf }}$ promotes the activity of p53, which induces cell cycle arrest and/or apoptosis. We have had a particular interest in understanding why the quality of lymphoid progenitors is reduced with age, and recent studies have shown that both Ink $4 a$ and Arf expression increases in pro-B cells from old mice and that inhibiting their expression can partially reverse the effects of aging (81). We also observed increased expression of Ink4a, but not Arf, in ETPs and their DN progeny (82).

Other age-associated changes may be more specific to the hematopoietic system. For example, altered expression of various B lineage transcription factors has been proposed to underlie declines in B lymphopoiesis (83). Recent interest has focused on BATF, the expression of which is highest in the hematopoietic system, in the decline of lymphoid-biased HSCs during aging. Wang and colleagues demonstrated that BATF levels increased in HSCs in response to DNA damage and that this resulted in the differentiation of lymphoid-biased HSCs at the expense of their self-renewal (27). This is a particularly interesting finding, because it provides a molecular mechanism for the age-related decline in the number of lymphoid-biased HSCs discussed above.

Although there has been considerable focus on stem and progenitor cells, aging also affects patterns of gene expression in mature $\mathrm{B}$ and $\mathrm{T}$ cells. As previously discussed, class switch recombination is impaired in aging murine and human B cells due to decreased expression of enzymes such as $\operatorname{AID}(53,84)$. Aberrant $\mathrm{T}$ cell signaling has also been associated with aging $(48,85)$, and the list of dysregulated genes continues to grow, as indicated by several recent reports (86-88), two of which focused on altered expression of dual specific phosphatases (DUSPs). DUSPs deactivate target kinases, including those in the MAPK pathway whose activity is critical for $T$ cell activation, differentiation, and cytokine production. One study reported the induction and sustained transcription of DUSP4 in activated CD4 ${ }^{+}$memory T cells from humans 65 and older, which correlated with their altered cytokine production and an impaired ability to provide help for B cells (87). A subsequent report from the same laboratory described the increased expression of DUSP 6 in naive $\mathrm{CD}^{+}{ }^{+}$human $\mathrm{T}$ cells, which resulted in activation of a lower fraction of Th cells, particularly in response to low-affinity stimuli (88).

These signaling abnormalities in HSCs, lymphoid progenitors, and mature B and T cells may occur, at least in part, as a result of the age-related alterations in the systemic and local tissue environments discussed above. For example, over time exposure to an inflammatory milieu might induce epigenetic modifications that affect the expression of genes required for growth, survival, or differentiation $(89,90)$. The observation that changes in methylation status as a result of differential expression of various DNA methyltransferases (DNMTs) can affect HSC behavior is consistent with this view. In this regard, loss of DNMT3a impairs differentiation of HSCs while their number increases in the bone marrow (91), and mice with hypomorphic expression of DNMT1 exhibit relatively normal myeloerythroid potential but show a significant block in lymphoid development (92). Nevertheless, cell-autonomous events 


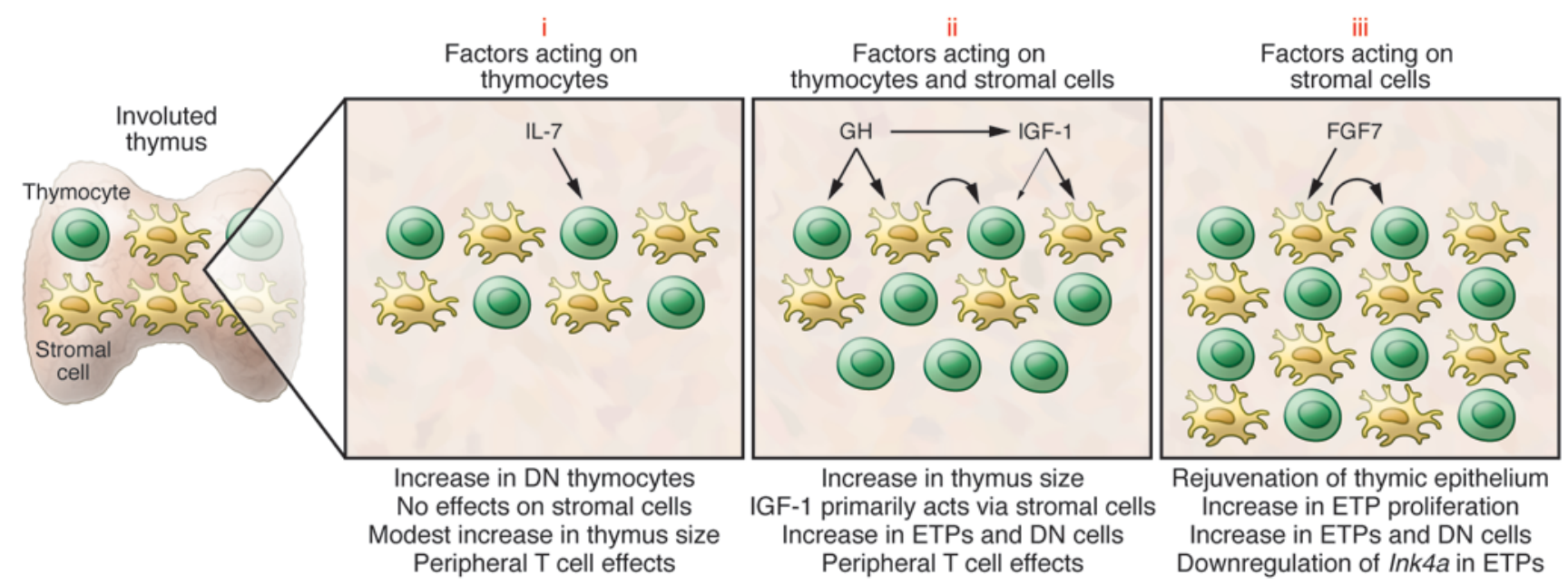

Figure 3

Selected strategies to rejuvenate the involuted thymus. The potential of several hormones and growth factors to rejuvenate the involuted thymus has been tested in various preclinical and clinical trials. Many of these factors can be grouped into three categories. Those in the first (i), such as IL-7, bind to progenitors in the bone marrow and thymus and have only modest effects on thymopoiesis. There is little evidence that IL-7 has effects on thymic stromal cells. Instead, the benefit of IL-7 may lie in its ability to stimulate peripheral T cell survival/expansion. The second category (ii) includes hormones such as GH that have been demonstrated in preclinical and clinical trials to stimulate thymopoiesis and increase thymic size. Many GH effects are mediated through induction of IGF-1. IGF-1 can bind to receptors on thymic stroma and thymocytes, although its actions are primarily mediated through effects on the former cells. Stromal cell-derived factors presumably then act on thymocytes (curved arrow). A third category of factors (iii), typified by FGF7, bind to stromal cells but not thymocytes. Stromal cell-induced factors then act on thymocytes (curved arrow), and we have recently demonstrated that effects include downregulation of Ink4a in ETPs (82). The thymopoietic effects of several additional factors have been evaluated, and recent detailed reviews should be consulted for more information $(99,100)$.

might also contribute to aging. Thus, repeated cell division may result in progressive telomere shortening that in turn results in DNA damage that can no longer be repaired efficiently due to agerelated defects in DNA repair mechanisms (75). It is interesting that telomere dysfunction has been associated with changes in mitochondrial metabolism (93), in view of a study linking compromised mitochondrial function and depressed thymopoiesis (94).

\section{Rejuvenating the aging immune system}

The ultimate goal of research in immune system aging is to use the information to develop strategies to stimulate immunity in the elderly (summarized in Table 1). As discussed above, a number of molecules have been identified whose aberrant expression contributes to aging, and targeting these molecules may potentially slow or reverse the aging process. For example, pharmacologic inhibition of Cdc42, a small Rho GTPase whose increased expression has been linked to murine HSC aging, with a selective Cdc42 activity inhibitor rejuvenated HSCs (95), and other studies have shown the potential of antioxidants to improve the function of old HSCs (76, 96). As additional age-related changes in the expression of transcription factors, signaling intermediates, cell cycle regulators, and microRNAs $(97,98)$ are defined, novel pharmacologic interventions will likely emerge. In this regard, the increased expression of DUSP6 in naive CD4 $4^{+} \mathrm{T}$ cells described above was due to the age-related decline in miR-181a expression (88).

Because of the key role played by $\mathrm{T}$ lymphocytes in the immune response and the clear effect of aging on mature $\mathrm{CD}^{+}$and $\mathrm{CD}^{+}$ cells, rejuvenation of the involuted thymus to replenish the peripheral $\mathrm{T}$ cell pool has been of considerable interest. A complete discussion of the various hormones and growth factors involved has been reviewed elsewhere $(99,100)$, but there has been considerable interest in using classical endocrine hormones such as GH and IGF-1 as well as other agents such as IL-7 and FGF7 to stimulate thymopoiesis (Figure 3).

GH and IGF-1 bind to receptors expressed on thymocytes (101) and thymic epithelial cells (102), and numerous studies (42) have shown that thymus size is increased in old mice administered either hormone $(101,103)$. These preclinical data were the basis for a human clinical trial to determine whether one year of daily $\mathrm{GH}$ administration could rejuvenate the thymus of HIV-infected adults. Despite low viral loads, those enrolled in this trial had low $\mathrm{CD}^{+} \mathrm{T}$ cell counts. GH treatment resulted in increased thymic mass, increased thymic output as measured by $\mathrm{T}$ cell receptor rearrangement excision circles in circulating $\mathrm{T}$ cells, and increased numbers of naive $\mathrm{CD}^{+} \mathrm{T}$ cells. Many GH effects are mediated through induction of IGF-1 production, and the elevated levels of IGF-1 in GH-treated patients were consistent with this. Administration of IGF-1 to old mice enhances thymopoiesis predominantly through effects on thymic epithelial cells and not thymocytes (104). The latter observations suggest that GH in the human subjects resulted in an induction of IGF-1 that functioned in a similar manner. It is important to note that the effects of $\mathrm{GH}$ were not thymus specific, as the subjects also had expanded numbers of naive peripheral $\mathrm{CD}^{+} \mathrm{T}$ cells (105).

In contrast to GH and IGF-1, which increase thymus cell number approximately two-fold in mice (101), FGF7 is particularly remarkable because its administration to old mice results in a renewal of the thymic microenvironment, increased numbers of ETPs, and a restoration of thymus cellularity to levels observed in the young $(106,107)$. FGF7, also known as keratinocyte growth factor and marketed as Kepivance (palifermin; Biovitrum), is approved by the FDA for treatment of oral mucositis in patients undergoing 
myelotoxic therapy. We hypothesized that the increased thymopoiesis in FGF7-treated old mice occurred because signals from the rejuvenated microenvironment in turn reduced Ink4a expression in ETPs. As predicted, levels of Ink4a expression in ETPs and DN cells harvested from FGF7-treated mice were downregulated, and these cells exhibited enhanced proliferation (82). These observations are consistent with a recent report showing that conditional deletion of Ink4a in DN thymocytes can significantly delay thymic involution (108). It will thus be of interest to identify the factor(s) released from FGF7-stimulated thymic epithelial cells that then act on aged ETPs and DN cells, as these could have therapeutic potential.

Strategies that focus on mature B and T cells may also be of value in stimulating immunity in the elderly. For example, if naive lymphocytes are unable to lodge efficiently in the spleen because niches are occupied by memory cells, deletion of the latter cells might create space. The depletion of mature lymphocytes might have feedback effects on primary lymphoid organs, as abrogation of mature B cells in aging mice has been reported to stimulate primary bone marrow B lymphopoiesis (109). It may also be possible to target specific pathways in old lymphocytes. For example, inhibition of mTOR, a PI3K pathway intermediate, with rapamycin has been shown to stimulate $\mathrm{CD} 8^{+} \mathrm{T}$ cell responses in preclinical studies (110). Targeting the expression of DUSP4 (87) or DUSP6 (88) in old memory or naive $\mathrm{CD}^{+} \mathrm{T}$ cells, respectively, may also have therapeutic potential. In support of this, the reduction of DUSP6 levels by increasing miR-181a expression or repressing activity with the allosteric inhibitor $\mathrm{BCI}$ improved the responsiveness of old naive $\mathrm{CD}^{+} \mathrm{T}$ cells (88).

Correcting age-related deficiencies in lymphocyte progenitors or mature $\mathrm{T}$ and $\mathrm{B}$ cells may result in significant restoration of immune function in the elderly. However, these cells would still reside in an aged microenvironment that could eventually dampen their potential to mature or function. Consistent with this view, Haynes and colleagues (111) found that $\mathrm{CD}^{+} \mathrm{T}^{+}$cells generated from old HSCs were functional in young but not old hosts, implying that the aged thymic or peripheral microenvironments critically influence the degree to which immune system rejuvenation can occur. In view of this point, optimal interventions may need to address the effects of aging on multiple cellular targets.

Approaches to inhibit or reverse aging should be widely available and applicable to a large cohort. In addition to pharmacologic interventions, caloric restriction $(\mathrm{CR})$ may also meet these criteria. CR has been reported to have multiple beneficial effects on the immune system of both rodents and non-human primates that include a delay in the accumulation of senescent T cells (112) and a stimulation of thymopoiesis (113). This latter effect is at first puzzling, because CR reportedly reduces IGF-1 secretion (114), and low levels of IGF-1 have, as discussed above, been associated with thymic involution. However, CR may also increase GH levels (114), which could be thymopoietic, or may work through IGF-1-independent pathways. For example, CR has been reported to block the age-related elevation of the thymic proadipogenic master regulator, PPAR $\gamma$ (113). In addition to further studies to increase our understanding of how $\mathrm{CR}$ affects the immune system, we also need to learn more about when it should commence in view of a report that beneficial effects were observed when it commenced in adults but were not evident when initiated in prepubertal or aged non-human primates (112). A final point is that, while CR is particularly attractive because it is a non-invasive approach, getting a large number of individuals to adopt this regimen might be challenging.

\section{Caveats in immune system rejuvenation}

The studies reviewed above provide proof-of-concept data that pharmacologic stimulation of immunity in the elderly is possible, and suggest that such interventions could be administered to a large cohort of individuals. However, as the field moves forward, there are several points to consider. First, how long do agents need to be administered, and how much rejuvenation is necessary in order to observe positive effects on immune function? These issues are relevant to the use of GH, IGF-1, and FGF7 to stimulate the thymus, because their effects are transient and the thymus reverts to its former size following the cessation of treatment. Another caveat is that factors such as GH, IGF-1, and FGF7 have widespread systemic effects due to the expression of their receptors on cells in multiple tissues. A concern with the use of some agents is that neoplastic cells might also express receptors resulting in tumor growth (115). Thus, like any drug, the use of these hormones to rejuvenate the thymus must be balanced with potential adverse effects. It will also be crucial to ensure that the rejuvenated immune system functions normally. In particular, it will be important to establish that the effector populations that develop have been appropriately tolerized and are not autoreactive. The fact that many autoimmune diseases occur in late adulthood makes this a particularly important issue (116).

\section{Conclusions and perspectives}

As indicated above, a tremendous amount is now known regarding the cellular changes that occur in the aging immune system and the molecular events that underlie them. Thus, it is not unreasonable to expect that this information will continue to be translated into therapies to rejuvenate the aging immune system.

It is likely that the effects of aging on the immune system will not be uniform between individuals. Thus, an ultimate goal would be to identify key biomarkers and establish simple laboratory tests to define each person's aging profile. Such information could then be used to develop a personalized approach in which targeted interventions could be directed to the individual's specific aging deficit. However, whatever standard of care is ultimately adopted for stimulating immunity, the emphasis should not necessarily be placed on increasing life span. Rather, the aim is to increase health span, defined as years of healthy living (117).

\section{Acknowledgments}

Work from the authors' laboratory was supported by NIH grant AG034875.

Address correspondence to: Kenneth Dorshkind, David Geffen School of Medicine at UCLA, 10833 Le Conte Avenue, Los Angeles, California 90095, USA. Phone: 310.206.9535; Fax: 310.206.9391; E-mail:kdorshki@mednet.ucla.edu.
1. Thompson W. Mortality associated with influenza
and respiratory syncytial virus in the United States.
JAMA. 2003;289(2):179-186.
2. McElhaney JE, Dutz JP. Better influenza vaccines
for older people: what will it take? J Infect Dis. 2008;
198(5):632-634.

3. Reichert T, Simonsen L, Sharma A, Pardo S, Fedson $\mathrm{D}$, Miller M. Influenza and the winter increase in mortality in the United States. Am J Epidemiol. 2004; 160(5):492-502.
4. Fleming D, Elliot A. The impact of influenza on the health and health care utilisation of elderly people. Vaccine. 2005;23:S1-S9.

5. Hardy RR, Kincade PW, Dorshkind K. The protean nature of cells in the B lymphocyte lineage. 
Immunity. 2007;26(6):703-714.

6. Love P, Bhandoola A. Signal integration and crosstalk during thymocyte migration and emigration. Nat Rev Immunol. 2011;11(7):469-477.

7. Rothenberg E. T cell lineage commitment: identity and renunciation. J Immunol. 2011; 186(12):6649-6655

8. Linton PJ, Dorshkind K. Age-related changes in lymphocyte development and function. Nat Immunol. 2004;5(2):133-139.

9. Lynch H, Goldberg G, Chidgey A, Van den Brink M, Boyd R, Sempowski G. Thymic involution and immune reconstitution. Trends Immunol. 2009;30(7):366-373.

10. Steinmann GG. Changes in the human thymus during aging. Curr Top Pathol. 1986;75:43-48.

11. Rossi DJ, et al. Cell intrinsic alterations underlie hematopoietic stem cell aging. Proc Natl Acad Sci US A. 2005;102(26):9194-9199.

12. Zediak VP, Maillard I, Bhandoola A. Multiple prethymic defects underlie age-related loss of $\mathrm{T}$ progenitor competence. Blood. 2007;110(4):1161-1167.

13. Taub D, Murphy W, Longo D. Rejuvenation of the aging thymus: growth hormone-mediated pathways and ghrelin-mediated signaling pathways. Curr Opin Pharmacol. 2010;10(4):408-424.

14. Song $Z$, et al. Alterations of the systemic environment are the primary cause of impaired $\mathrm{B}$ and $\mathrm{T}$ lymphopoiesis in telomere-dysfunctional mice. Blood. 2010;115(8):1481-1489.

15. de Haan G, Van Zant G. Dynamic changes in mouse hematopoietic stem cell numbers during aging. Blood. 1999;93(10):3294-3301.

16. Harrison D, Astle C, Stone M. Numbers and functions of transplantable primitive immunohematopoietic stem cells. Effects of age. J Immunol. 1989; 142(11):3833-3840.

17. Pang W, et al. Human bone marrow hematopoietic stem cells are increased in frequency and myeloidbiased with age. Proc Natl Acad Sci U S A. 2011; 108(50):20012-20017.

18. Sudo K, Ema H, Morita Y, Nakauchi H. Age-associated characteristics of murine hematopoietic stem cells. J Exp Med. 2000;192(9):1273-1280.

19. Cho R, Sieburg H, Muller-Sieburg C. A new mechanism for the aging of hematopoietic stem cells: aging changes the clonal composition of the stem cell compartment but not the individual stem cells. Blood. 2008;111(12):5553-5561.

20. Muller-Sieburg C, Cho R, Karlsson L, Huang J, Sieburg H. Myeloid-biased hematopoietic stem cells have extensive self-renewal capacity but generate diminished lymphoid progeny with impaired IL-7 responsiveness. Blood. 2004;103(11):4111-4118.

21. Challen G, Boles N, Chambers S, Goodel M. Distinct hematopoietic stem cells subtypes are differentially regulated by TGF- $\beta 1$. Cell Stem Cell. 2010; 6(3):265-278.

22. Morita Y, Ema H, Nakauchi H. Heterogeneity and hierarchy within the most primitive hematopoietic stem cell compartment. J Exp Med. 2010; 207(6):1173-1182.

23. Beerman I, et al. Functionally distinct hematopoietic stem cells modulate hematopoietic lineage potential during aging by a mechanism of clonal expansion. Proc Natl Acad Sci U S A. 2010; 107(12):5465-5470.

24. Dykstra B, Olthof S, Schreuder J, Ritsema M, De Haan G. Clonal analysis reveals multiple functional defects of aged murine hematopoietic stem cells. J Exp Med. 2011;208(13):2691-2703.

25. Solana R, Tarazona R, Gayoso I, Lesur O, Dupuis G, Fulop T. Innate immunosenescence: Effect of aging on cells and receptors of the innate immune system in humans. Semin Immunol. 2012;24(5):331-341.

26. Shaw A, Joshi S, Greenwood H, Panda A, Lord J. Aging of the innate immune system. Curr Opin Immunol. 2010;22(4):507-513.
27. Wang J, et al. A differentiation checkpoint limits hematopoietic stem cell self-renewal in respnse to DNA damage. Cell. 2012;148(5):1001-1014.

28. Miller JP, Allman D. The decline in B lymphopoiesis in aged mice reflects loss of very early B-lineage precursors. J Immunol. 2003;171(5):2326-2330.

29. Min H, Montecino-Rodriguez E, Dorshkind K. Effects of aging on the common lymphoid progenitor to pro-B cell transition. J Immunol. 2006; 176(2):1007-1012.

30. Sherwood EM, Xu W, King AM, Blomberg BB, Riley RL. The reduced expression of surrogate light chains in B cell precursors from senescent BALB/C mice is associated with decreased E2A proteins. Mech Ageing Develop. 2000;118(1-2):45-59.

31. Johnson KM, Owen K, Witte PL. Aging and developmental transitions in the B cell lineage. Int Immunol. 2002;14(11):1313-1323.

32. Min H, Montecino-Rodriguez E, Dorshkind K. Reduction in the developmental potential of intrathymic $\mathrm{T}$ cell progenitors with age. J Immunol. 2004;173(1):245-250.

33. Heng TSP, Goldberg GL, Gray DHD, Sutherland JS, Chidgey AP, Boyd RL. Effects of castration on thymocyte development in two different models of thymic involution. J Immunol. 2005;175(5):2982-2993.

34. Min H, Montecino-Rodriguez E, Dorshkind K. Effects of aging on early B- and T-cell development. Immunol Rev. 2005;205:7-17.

35. Allman D, et al. Thymopoiesis independent of common lymphoid progenitors. Nat Immunol. 2003; 4(2):168-174

36. Labrie JE, Sah AP, Allman DM, Cancro MP, Gerstein RM. Bone marrow microenvironmental changes underlie reduced RAG-mediated recombination and $\mathrm{B}$ cell generation in aged mice. J Exp Med. 2004;200(4):411-423.

37. Stephan RP, Reilly CR, Witte PL. Impaired ability of bone marrow stromal cells to support B lymphopoiesis. Blood. 1998;91(1):75-88.

38. Manley N, Richie E, Blackburn C, Condie B, Sage J. Structure and function of the thymic microenvironment. Front Biosci. 2011;17:2461-2477.

39. Chinn IK, Blackburn CC, Manley NR, Sempowski GD. Changes in primary lymhoid organs with aging. Semin Immunol. 2012;24(5):309-320.

40. Dixit V. Impact of immune-metabolic interactions on age-related thymic demise and $\mathrm{T}$ cell senescence. Semin Immunol. 2012;24(5):321-330.

41. Naveiras O, Nardi V, Wenzel P, Hauschka P, Fahey F, Daley GQ. Bone-marrow adipocytes as negative regulators of the hematopoietic microenvironment. Nature. 2009;460(7252):259-264.

42. Dorshkind K, Horseman ND. The roles of prolactin, growth hormone, insulin-like growth factor-I, and thyroid hormones in lymphocyte development and function: insights from genetic models of hormone and hormone receptor deficiency. Endocr Rev. 2000;21(3):292-312.

43. Lamberts S, van den Beld A, van der Lely A. The endocrinology of aging. Science. 1997; 278(5337):419-424.

44. Jamieson BD, et al. Generation of functional thymocytes in the human adult. Immunity. 1999; 10(5):569-575.

45. Nikolich-Žugich J, Li G, Uhrlaub JL, Renkema KR, Smithey MJ. Age-related changes in CD8 T cell homeostasis and immunity to infection. Semin Immunol. 2012;24(5):356-364.

46. Clambey E, White J, Kappler J, Marrack P. Identification of two major types of age-associated CD8 clonal explansions with highly divergent properties. Proc Natl Acad Sci U S A. 2008; 105(35):12997-13002.

47. Effros RB. Loss of CD28 expression on T lymphocyes: a marker of replicative senescence. Dev Comp Immunol. 1997;21(6):471-478.

48. Goronzy JJ, Li G, Yu M, Weyand CM. Signaling pathways in aged $\mathrm{T}$ cells-a reflection of $\mathrm{T}$ cell differentiation, cell senescence and host environment. Semin Immunol. 2012;24(5):365-372.

49. Strindhall J, et al. No immune risk profile among individuals who reach 100 years of age: findings from the Swedish NONA immune longitudinal study. Exp Gerontol. 2007;42(8):753-761.

50. McElhaney J, et al. The unmet need in the elderly: how immunosenescence, CMV infection, co-morbidities and frailty are a challenge for the development of more effective influenza vaccines. Vaccine. 2012;30(12):2060-2067.

51. Frasca D, Blomberg BB. Effects of aging on B cell function. Curr Opin Immunol. 2009;21(4):425-430.

52. Shi Y, Yamazaki T, Okubo Y, Uehara Y, Sugane K, Agematsu K. Regulation of aged humoral immune defense against pneumococcal bacteria by IgM memory B cell. J Immunol. 2005;175(5):3262-3267.

53. Frasca D, et al. Aging down-regulates the transcription factor E2A, activation-induced cytidine deaminase, and Ig class switch in human B cells. J Immunol. 2008;180(8):5283-5290.

54. Miller JP, Cancro MP. B cells and aging: balancing the homeostatic equation. Exp Gerontol. 2007; 42(5):396-399.

55 . Sasak S, et al. Limited efficacy of inactivated influenza vaccine in elderly individuals is associated with decreased production of vaccine-specific antibodies. J Clin Invest. 2011;121(8):3109-3119.

56. Usinger W, Lucas A. Avidity as a determinant of the protective efficacy of human antibodies to pneumoccal capsular polysaccharides. Infect Immun. 1999;67(5):2366-2370.

57. Westerink M, Schroeder HJ, Nahm M. Immune responses to pneumococcal vaccines in children and adults: rationale for age-specific vaccination. Aging Dis. 2012;3(1):51-67.

58. Romero-Steiner $\mathrm{S}$, et al. Reduction in functional antibody activity against Streptococcus pneumoniae in vaccinated elderly individuals highly correlates with decreased IgG antibody avidity. Clin Infect Dis. 1999;29(2):281-288.

59. Waffarn E, Baumgarth N. Protective B cell responses to flu-no fluke! J Immunol. 2011; 186(7):3823-3829.

60. Birjandi S, Ippolito J, Ramadorai A, Witte PL. Alterations in marginal zone macrophages and marginal zone B cells in old mice. J Immunol. 2011;186(6):3441-3451.

61. Baumgarth N. The double life of a B-1 cell: selfreactivity selects for protective effector functions. Nat Rev Immunol. 2011;11(1):34-46.

62. Haas KM, Poe JC, Steeber DA, Tedder TF. B-1a and $\mathrm{B}-1 \mathrm{~b}$ cells exhibit distinct developmental requirements and have unique functional roles in innate and adaptive immunity to S. pneumoniae. Immunity. 2005;23(1):7-18.

63. Griffin DO, Holodick NE, Rothstein TL. Human B1 cells in umbilical cord and adult peripheral blood express the novel phenotype CD20 ${ }^{+} \mathrm{CD} 27^{+}$ CD43+ CD70-. J Exp Med. 2011;208(1):67-80.

64. Franceschi $C$, et al. Inflammaging and anti-inflammaging: a systemic perspective on aging and longevity emerged from studies in humans. Mech Ageing Develop. 2007;128(1):92-105.

65. Coppe J-P, Desprez P-Y, Krtolica A, Campisi J. The senescence-associated secretory phenotype: the dark side of tumor suppression. Annu Rev Pathol. 2010;5:99-118.

66. Cavanagh M, Weyand C, Goronzy J. Chronic inflammation and aging: DNA damage tips the balance. Curr Opin Immunol. 2012;24(4):488-493.

67. Rodier F, et al. Persistent DNA damage signalling triggers senescence-associated inflammatory cytokine secretion. Nat Cell Biol. 2009;11(8):973-979.

68. Effros RB. Role of T lymphocyte replicative senescence in vaccine efficacy. Vaccine. 2007;25(4):599-604. 69. Weng N, Akbar A, Gornzy J. CD28(-) T cells: their 
role in the age-associated decline of immune function. Trends Genet. 2009;30(7):306-312.

70. Maue AC, Yager E, Swain S, Woodland D, Blackman M, Haynes L. T-cell immunosenescence: lessons learned from mouse models of aging. Curr Opin Immunol. 2009;30(7):301-305.

71. Larbi A, Pawelec G, Wong SC, Goldeck D, Tai JJ, Fulop T. Impact of age on T cell signaling: a general defect or specific alterations? Ageing Res Rev 2011;10(3):370-378.

72. Agrawal A, Gupta S. Impact of aging on dendritic cell functions in humans. Ageing Res Rev. 2011;10(3):336-345.

73. Huang MC, Liao JJ, Bonasera S, Longo DL, Goetzl EJ. Nuclear factor-kappaB-dependent reversal of aging-induced alterations in $\mathrm{T}$ cell cytokines. FASEB J. 2008;22(7):2142-2150.

74. Schraml B, et al. The AP-1 transcription factor Batf controls $\mathrm{T}(\mathrm{H}) 17$ differentiation. Nature. 2009;460(7253):405-409.

75. Sahin E, DePinho RA. Linking functional declines of telomeres, mitochondira and stem cells during ageing. Nature. 2010;464(7288):520-528.

76. Tothova Z, Gilliland DG. FoxO transcription factors and stem cell homeostasis: insights from the hematopoietic system. Cell Stem Cell. 2007;1(2):140-152.

77. Feng Z, Hu W, Teresky A, Hernando E, CordonCardo C, Levine A. Declining p53 function in the aging process: a possible mechanism for the increased tumor incidence in older populations. Proc Natl Acad Sci US A. 2007;104(42):16633-16638.

78. Ito K, et al. Reactive oxygen species act through p38 MAPK to limit the lifespan of hematopietic stem cells. Nat Med. 2006;12(4):446-451.

79. Krishnamurthy J, et al. Ink $4 a / A r f$ expression is a biomarker of aging. J Clin Invest. 2004;114(9):1299-1307.

80. Sharpless NE. Ink4a/Arf links senescence and aging. Exp Gerontol. 2004;39(11-12):1751-1759.

81. Signer RAJ, Montecino-Rodriguez E, Witte ON, Dorshkind K. Aging and cancer resistance in lymphoid progenitors are linked processes conferred by $16^{\text {Ink4a }}$ and Arf. Genes Dev. 2008; 22(22):3115-3120

82. Berent-Maoz B, Montecino-Rodriguez E, Signer RA, Dorshkind K. Fibroblast growth factor-7 partially reverses murine thymocyte progenitor aging by repression of Ink4a. Blood. 2012;119(24):5715-5721.

83. Frasca D, Nguyen D, Riley R, Blomberg B. Decreased E12 and/or E47 transcription factor activity in the bone marrow as well as in the spleen of aged mice. J Immunol. 2003;170(2):719-726.

84. Frasca D, Landin AM, Riley RL, Blomberg B. Mechanisms for decreased function of $B$ cells in aged mice and humans. IImmunol. 2008;180(5):2741-2746.

85. Garcia G, Miller R. Age-related defects in the cyto- skeleton signaling pathways of CD4 T cells. Ageing Res Rev. 2011;10(1):26-34.

86. Long D, et al. Single-cell network profiling of peripheral blood mononuclear cells from healthy donors reveals age- and race-associated differences in immune signaling pathway activation. J Immunol. 2012;188(4):1717-1725

87. Yu M, et al. Signal inhibition by the dual-specific phosphatase 4 impairs T cell-dependent B-cell responses with age. Proc Natl Acad Sci U S A. 2012; 109(15):879-888.

88. Li G, et al. Decline in miR-181a expression with age impairs $T$ cell receptor sensitivity by increasing DUSP6 activity. Nat Med. 2012;18(10):1518-1524.

89. Berdasco M, Esteller M. Hot topics in epigenetic mechanisms of aging: 2011. Aging Cell. 2012; 11(2):181-186

90. Rando T, Chang H. Aging, rejuvenation, and epigenetic reprogramming: resetting the aging clock. Cell. 2012;148(1-2):46-57.

91. Challen G, et al. Dnmt3a is essential for hematopoietic stem cell differentiation. Nat Genet. 2011; 44(1):23-31.

92. Broske A, et al. DNA methylation protects hematopoietic stem cell multipotency from myeloerythorid restriction. Nat Genet. 2009;41(11):1207-1215.

93. Sahin E, et al. Telomere dysfunction induces metabolic and mitochondrial compromise. Nature. 2011; 470(7334):359-365.

94. Liu J, et al. Bmi1 regulates mitochondrial function and the DNA damage response pathway. Nature. 2009;459(7245):387-392.

95. Florian $\mathrm{M}$, et al. Cdc42 activity regulates hematopoietic stem cell aging and rejuvenation. Cell Stem Cell. 2012;10(5):520-530.

96. Ito K, et al. Regulation of oxidative stress by ATM is required for self-renewal of hematopoietic stem cells. Nature. 2004;431(7011):997-1002.

97. Hammond SM, Sharpless NE. HMGA2, microRNAs, and stem cell aging. Cell. 2008;135(6):1013-1016

98. Gorospe M, Abdelmohsen A. MicroRegulators come of age in senescence. Trends Genet. 2011; 27(6):233-241.

99. Hollander GA, Krenger W, Blazar B. Emerging strategies to boost thymic function. Curr Opin Pharmacol. 2010;10(1):1-11.

100. Holland A, Van den Brink MRM. Rejuvenation of the aging $\mathrm{T}$ cell compartment. Curr Opin Immunol. 2009;21(4):454-459.

101. Montecino-Rodriguez E, Clark R, Dorshkind K Effects of insulin-like growth factor administration and bone marrow transplantation on thymopoiesis in aged mice. Endocrinology. 1998;139(10):4120-4126.

102. Kermani H, et al. Expression of the growth hormone/insulin-like growth factor axis during Balb/c thymus ontogeny and effects of growth hormone upon ex vivo T cell differentiation. Neuroimmunomodulation. 2012;19(3):137-147.

103. Welniak L, Sun R, Murphy W. The role of growth hormone in T cell development and reconstitution. J Leuk Biol. 2002;71(3):381-387.

104. Chu Y, et al. Exogenous insulin-like growth factor 1 enhances thymopoiesis predominantly through thymic epithelial cell expansion. Blood. 2008;112(7):2836-2846.

105. Napolitano LA, et al. Growth hormone enhances thymic function in HIV-1-infected adults. J Clin Invest. 2008;118(3):1085-1098.

106. Min D, Panoskaltsis-Mortari A, Kuro-O M, Holländer GA, Blazar BR, Weinberg KI. Sustained thymopoiesis and improvement in functional immunity induced by exogenous KGF administration in murine models of aging. Blood. 2007;109(6):2529-2537.

107. Rossi SW, et al. Keratinocyte growth factor (KGF) enhances postnatal T-cell development via enhancements in proliferation and function of thymic epithelial cells. Blood. 2007;109(9):3803-3811.

108. Liu Y, et al. Expression of p16Ink4a prevents cancer and promotes aging in lymphocytes. Blood. 2011;117(12):3257-3267.

109. Keren Z, et al. B-cell depletion reactivates B lymphopoiesis in the $\mathrm{BM}$ and rejuvenates the $\mathrm{B}$ lineage in aging. Blood. 2011;117(11):3104-3112.

110.Chi H. Regulation and function of mTOR signalling in T cell fate decisions. Nat Rev Immunol. 2012;12(5):325-338.

111.Eaton SM, Maue AC, Swain SL, Haynes L. Bone marrow precursor cells from aged mice generate CD4 $\mathrm{T}$ cells that function well in primary and memory responses. J Immunol. 2008;181(7):4825-4831.

112. Messaoudi I, et al. Optimal window of caloric restriction onset limits its beneficial impact on T-cell senescence in primates. Aging Cell. 2008; 7(6):908-919.

113.Yang $H$, Youm $Y$, Dixit V. Inhibition of thymic adipogenesis by caloric restriction is coupled with reduction in age-related thymic involution. J Immunol. 2009;183(5):3040-3052.

114.Sonntag W, et al. Pleiotroic effects of growth hormone and insulin-like growth factor (IGF)-1 on biological aging: inferences from moderate caloric-restricted animals. J Gerontol A Biol Sci Med Sci. 1999;54(12):B521-B538.

115. Pollak M. Insulin and insulin-like growth factor signalling in neoplasia. Nat Rev Cancer. 2008; 8(12):915-928.

116. Goronzy JJ, Weyand CM. Immune aging and autoimmunity. Cell Mol Life Sci. 2012;69(10):1615-1623.

117. Dorshkind K, Montecino-Rodriguez E, Signer RA. The ageing immune system: is it ever too old to become young again? Nat Rev Immunol. 2009; 9(1):57-62. 